\title{
Dégager les besoins en soins de soutien des femmes atteintes d'un cancer ovarien
}

par D $\mathrm{D}^{\mathrm{re}}$ Margaret I. Fitch et $\mathrm{D}^{\mathrm{re}}$ Rose Steele

\section{Abrégé}

Les femmes diagnostiquées d'un cancer de l'ovaire subissent les nombreuses conséquences à court et à long terme du cancer et de son traitement. L'impact du cancer ne se limite pas à la sphère physique et pourtant, il existe fort peu d'information sur les types de besoins de ces femmes et si elles souhaitent ou non recevoir de l'aide afin de répondre à leurs besoins.

Le but principal de cette étude descriptive transversale était de cerner les besoins en soins de soutien (physiques, affectifs, sociaux, informationnels, spirituels, psychologiques et pratiques) des femmes atteintes d'un cancer ovarien qui fréquentaient en ambulatoire un centre de cancérologie intégré. Un but additionnel de l'étude était de déterminer si les femmes voulaient recevoir de l'aide afin de satisfaire ces besoins.

Cinquante femmes diagnostiquées d'un cancer de l'ovaire ont participé à cette étude en remplissant un questionnaire d'autoévaluation (enquête sur les besoins en soins de soutien). Les données indiquaient qu'un éventail de besoins en soins de soutien demeuraient insatisfaits chez ce groupe de patientes. Huit des dix besoins les plus fréquemment signalés étaient de nature psychosociale comme la crainte que le cancer ne revienne ou ne se propage. Les femmes ont également exprimé une gamme de difficultés sur le plan de la prise en charge de leurs besoins. Toutefois, cette réalité n'empêchait pas un nombre important de femmes d'indiquer qu'elles ne désiraient pas recevoir l'aide du personnel de la clinique relativement à certains de ces besoins. Des suggestions relatives à la pratique et aux recherches à réaliser à l'avenir sont offertes en vue d'aider les infirmières en oncologie à prodiguer les soins à ces femmes.

\section{Identifying supportive care needs of women with ovarian cancer}

\section{Abstract}

Women diagnosed with ovarian cancer may experience many short-term and long-term effects from cancer and its treatment. Cancer has more than a physical impact, yet there is a lack of information about the types of needs these women have and whether they want help in meeting their needs.

The main purpose of this cross-sectional, descriptive study was to identify the supportive care needs (physical, emotional, social, informational, spiritual, psychological and practical) of women with ovarian cancer who attended a comprehensive, outpatient cancer centre. A further purpose was to determine if women wanted assistance in meeting those needs.

A total of 50 women diagnosed with ovarian cancer participated in this study by completing a self-report questionnaire (The Supportive Care Needs Survey). The data indicated that a range of supportive care needs remained unmet for this patient group. Eight of the top 10 most frequently reported needs were psychosocial, such as fears about the cancer returning or spreading. The women also expressed a range of difficulty in managing their needs. However, despite this reality, significant numbers of women indicated they did not wish to have assistance from the clinic staff with some needs. Suggestions for practice and future research are offered to assist oncology nurses in providing care to these women.
Le cancer de l'ovaire est la quatrième cause de décès par cancer chez les Canadiennes. Selon les estimations, 2500 nouveaux cas de cancer de l'ovaire et 1700 décès dus à ce type de cancer étaient prévus en 2008 (Institut national du cancer du Canada, 2008). Il est possible que les femmes atteintes d'un cancer ovarien soient confrontées à des situations particulièrement pénibles et qu'elles éprouvent de la détresse du fait de l'intensité des procédures chirurgicales et de la toxicité des protocoles chimiothérapeutiques (Armstrong, 2002; Pignata, Ballatori, Favalli \& Scambia, 2001). On manque actuellement de connaissances sur les besoins en soins de soutien de ces femmes.

Le personnel infirmier de la clinique de gynécologie d'un centre de cancérologie intégré offrant des soins ambulatoires en Ontario, au Canada, souhaitait mieux comprendre les besoins en soins de soutien des femmes atteintes d'un cancer ovarien fréquentant sa clinique. Le personnel voulait prodiguer des soins de la plus haute qualité à ces femmes et approfondir ses connaissances relativement à leurs besoins insatisfaits. Quoique les infirmières aient une idée de ce dont les femmes avaient besoin, elles disposaient de fort peu de recherche empirique à l'appui de leurs croyances. L'étude a été conçue en vue de combler cette lacune.

\section{Revue de la littérature}

Le cancer de l'ovaire a une évolution grave et variable caractérisée par une réponse initiale aux traitements antinéoplasiques, suivie d'une rechute et de la progression de la maladie. Cela fait que cette maladie a une importante incidence physique et affective sur les femmes et leurs familles (Fitch, Deane \& Howell, 2003; Fitch, Gray \& Franssen, 2001; Howell, Fitch \& Deane, 2003a, 2003b). Certains chercheurs ont décrit son incidence sur la qualité de vie (Bezjak et al., 2004; Ersek, Ferrel, Dow \& Melancon, 1997; Ferrell et al., 2005; Fish \& Lewis, 1999; Lockwood-Rayermann, 2006; Schulman-Green, Ercolano, Dowd, Schwartz \& McCorkle, 2008; Wenzel et al., 2002), tandis que d'autres ont fait état du fardeau des symptômes (Montazeri, McEwen \& Gillis, 1996; Portenoy et al., 1994) et de la détresse psychosociale éprouvée par ce groupe de patientes (Greimel \& Friedl, 2000; Hamilton, 1999; Wenzel et al., 2002). Des résultats relativement uniformes ont été rapportés : une anxiété et une dépression marquées ont été observées chez de nombreuses femmes; la sexualité en subissait souvent un impact négatif; le fonctionnement social était perturbé, notamment en ce qui concerne l'aptitude à aller au travail; enfin, le fonctionnement physique et la progression de la maladie permettaient souvent de prévoir les changements touchant la qualité de vie. Les femmes signalant une moindre qualité de vie étaient plus susceptibles d'être plus jeunes, mieux instruites et/ou sans partenaire de vie ou d'avoir un cancer avancé (SchulmanGreen et al.). Il arrive fréquemment que les données relatives aux patientes porteuses d'un cancer de l'ovaire soient subsumées dans les études portant sur l'ensemble des cancers gynécologiques

\section{Au sujet des auteurs}

Dre Margaret I. Fitch, inf., Ph.D., Chef, Soins infirmiers en oncologie et Soins de soutien, Centre régional de cancérologie de Toronto-Sunnybrook. Courriel : marg.fitch@sunnybrook.ca

Dre Rose Steele, inf., Ph.D., Professeure, École de sciences infirmières, Faculty of Health, Université York, Toronto, ON. 
(Bodurka-Bevers et al., 2000; Ell et al., 2005; Norton et al., 2004; Steele \& Fitch, 2008; Tobano, Condosta \& Coons, 2002) et qu'elles ne soient donc pas isolées.

Le traitement du cancer de l'ovaire peut entraîner une morbidité chirurgicale et une toxicité chimiothérapeutique lesquelles sont associées à une neuropathie grave, à des altérations de l'image corporelle, à l'altération des relations avec le partenaire de vie ou d'autres êtres chers, des problèmes d'ordre sexuel et la perte de la fertilité (Hoskins et al., 2005; Jenkins, 1998). Le traitement chirurgical des cancers pelviens et l'oophorectomie ou l'ablation d'un ou des ovaires par radio/chimiothérapie peuvent entraîner la dyspareunie. Jusqu'à $50 \%$ des patientes traitées pour un cancer gynécologique éprouvent un type de problème sexuel ou un autre (Andersen \& Van Der Does, 1994). Les symptômes accompagnant les cancers gynécologiques et leurs traitements souvent invasifs incluent des changements défavorables de la libido ou de la satisfaction sexuelle. Ces symptômes ont été attribués aux changements affectant les tissus irradiés, à la ménopause prématurée, au rétrécissement du vagin ou à une modification de l'image corporelle (Hoskins et al.).

Dans une étude ethnographique basée sur la documentation relative à la correspondance entretenue pendant sept ans par des survivantes du cancer ovarien et à un bulletin de soutien, Ferrell, Smith, Cullinane et Melancon (2003) ont constaté que les effets secondaires débilitants du traitement étaient souvent pires que la maladie proprement dite. Le symptôme le plus courant était la douleur, notamment la neuropathie périphérique. Des problèmes gastrointestinaux tels que la nausée, la diarrhée et la constipation étaient également mentionnés à titre de sources de frustration. Les symptômes de la ménopause comme la sécheresse vaginale et les bouffées de chaleur, la perturbation des cycles menstruels et les problèmes liés à la fertilité étaient autant de sujets d'inquiétude pour de nombreuses femmes. Finalement, les femmes ont signalé une multitude d'autres symptômes qui avaient un impact négatif sur leur qualité de vie, p. ex. une perte auditive, l'ostéoporose, des problèmes de dentition, des affections cutanées, des perturbations de l'appétit et du sommeil. Une analyse plus poussée de ces données a révélé que les femmes faisaient appel à la spiritualité à la fois pour donner un sens à l'expérience et comme stratégie d'adaptation en vue d'exercer une incidence positive sur leur qualité de vie (Ferrell, Smith, Cullinane et al.).

Des symptômes tels que la fatigue et la douleur sont fréquemment associés au cancer de l'ovaire et ont un impact significatif sur la vie quotidienne. Dans l'étude de Ferrell, Smith, Juarez et Melancon (2003), la fatigue constituait un problème parce qu'elle réduisait l'aptitude des femmes à assumer leurs responsabilités journalières et que ces dernières jugeaient souvent qu'elles n'avaient pas suffisamment d'énergie pour soutenir leur famille. Selon Donovan et Ward (2005), 27 des 49 femmes participant à leur étude corrélationnelle descriptive ont défini la fatigue comme étant un symptôme pénible et incontrôlable. Plus de la moitié des femmes (59\%) n'avaient pas abordé le sujet de la fatigue avec leurs prestataires de soins lors de leur dernier rendez-vous et 56 \% déclaraient qu'aucun professionnel de la santé ne leur avait donné de conseils en matière de gestion de la fatigue. Donovan et Ward suggéraient que cette absence de conseils de la part des professionnels contribuait aux perceptions négatives des femmes. Portenoy et al. (1994) ont découvert que la douleur était courante, particulièrement chez les femmes porteuses d'un cancer avancé. Ces chercheurs utilisaient une échelle globale de la douleur dans leur enquête auprès de 151 femmes subissant un traitement pour leur cancer de l'ovaire. Plus de $50 \%$ des femmes de l'échantillon signalaient d'autres symptômes en sus de la douleur, notamment la fatigue, la détresse psychosociale et l'insomnie. La prise en charge d'une multitude de symp- tômes est un aspect important de la prestation des soins aux femmes sous traitement puisque celui-ci s'accompagne d'effets secondaires appréciables (Lockwood-Rayermann, 2006).

Browall, Carlsson et Horvath (2004) ont évalué les besoins d'information au moyen de neuf items chez un échantillon de 64 femmes ayant reçu un traitement pour le cancer ovarien. Les mesures ont été effectuées à trois reprises : 2-3 semaines après le diagnostic, à la fin du traitement primaire et enfin, six mois après celle-ci. Les besoins d'information sur la maladie et le traitement venaient en tête, et leur score restait plutôt constant lors des trois mesures. L'information sur les aspects psychosociaux et les soins auto-administrés obtenaient le score le plus faible. Jefferies (2002) a exploré les besoins d'information et les besoins affectifs de 24 patientes atteintes d'un cancer de l'ovaire. Elle a constaté que $96 \%$ des participantes avaient reçu des renseignements oraux et que presque deux tiers d'entre elles (66 \%) avaient reçu de l'information écrite. Toutefois, $31 \%$ des femmes estimaient que l'information écrite présentait de sérieuses lacunes. Le moment choisi pour la remise d'information, la quantité de cette dernière et la manière dont elle est fournie revêtaient de l'importance pour les participantes, et $71 \%$ d'entre elles étaient satisfaites du moment choisi, $83 \%$ de la quantité et $87 \%$ de la méthode de fourniture. Dans une étude qualitative, Howell et al. (2003a) rapportaient que les femmes atteintes d'un cancer de l'ovaire $(n=18)$ voulaient que leurs soins soient personnalisés et basés sur leurs propres perceptions du genre d'information qu'elles jugeaient utile d'avoir. Le maintien d'un dialogue et de conversations entre patients et prestataires de la santé était perçu comme étant essentiel, particulièrement dans les cas de cancer de l'ovaire récidivant.

La détresse psychosociale est une expérience courante chez les patientes ayant le cancer de l'ovaire, notamment durant la période faisant immédiatement suite au diagnostic. Zabora, Brintzenhofeszoc, Curbow, Hooker et Piantadosi (2001) mentionnaient qu'environ $20 \%$ de ces patientes éprouvent un niveau de détresse significativement plus élevé après un nouveau diagnostic. La détresse psychosociale a également été constatée chez les femmes lors du processus de dépistage du cancer de l'ovaire, durant la phase de diagnostic et de traitement de la maladie et lors des récidives (Kornblith et al., 1995; Petersen, Graham \& Qunlivan, 2005; Power, Brown \& Ritvo, 2008; Robinson, Rosen \& Bradley, 1997). De hauts taux d'anxiété et de dépression ont été signalés pendant le traitement de chimiothérapie (Guidozzi, 1993; Kornblith et al.), de hauts taux de détresse se poursuivant chez un tiers des survivants à long terme du cancer de l'ovaire (Ersek et al., 1997).

L'anxiété a également été documentée dans une étude qualitative explorant les perspectives des patientes sur leur diagnostic de cancer et sur leur traitement (Ekman, Bergbom, Ekman, Berthold \& Mahsneh, 2004). Dix Suédoises ont ainsi été interviewées au moment du diagnostic, durant la chimiothérapie et après l'achèvement de cette dernière. Les auteurs ont cerné la nécessité d'offrir du soutien à ces femmes alors qu'elles apprenaient à faire face à leur anxiété. Dans son étude, Jefferies (2002) a découvert que les femmes atteintes d'un cancer de l'ovaire recevaient un soutien affectif de diverses sources : membres de la famille, amies et professionnels de la santé. Les groupes de soutien étaient utiles puisqu'ils leur permettaient de parler avec des femmes vivant une situation similaire à la leur et que les membres se donnaient un soutien affectif mutuel. Cependant, les femmes n'étaient pas toutes amatrices des groupes de soutien, surtout lorsqu'un de ses membres tombait très malade ou même mourait.

Norton et ses collègues (2004) ont indiqué que le stress psychologique, mesuré sous forme d'anxiété, de dépression et de perte de contrôle des émotions/du comportement, se manifestait chez un grand nombre des femmes atteintes d'un cancer ovarien $(\mathrm{n}=143)$. Deux modèles à équations structurelles appuient leurs hypothèses à savoir que le contrôle et l'estime de soi perçus sont deux méca- 
nismes qui expliquent comment le stress lié à la maladie et le stress interpersonnel peuvent être associés au stress psychologique chez ces femmes. Norton et al. (2004) avançaient que la déficience physique mène au stress psychologique chez ces patientes parce qu'elle sape leur sentiment de maîtrise. Ils ont également découvert que les comportements dénotant un manque de compréhension de la part des membres de la famille et des amis entraînaient une estime de soi moindre et un plus haut niveau de stress psychologique. Il a été suggéré que des stratégies d'adaptation telles que chercher à obtenir des services de soutien adéquats peuvent modérer la détresse psychosociale éprouvée par les femmes porteuses d'un cancer gynécologique (Lutgendorf et al., 2002; Norton et al., 2005).

Il est clair qu'il faut prendre en compte les multiples dimensions des répercussions du cancer et de son traitement. Cependant, bien qu'il existe des travaux de recherche sur les effets physiques et psychosociaux du cancer ovarien chez les femmes, l'éventail élargi des besoins de cette population en matière de soins de soutien n'a pas encore été déterminé avec précision. En outre, on ne sait pas très bien si les femmes veulent recevoir de l'aide ou non en ce qui concerne ces besoins. Il convient donc de dégager les besoins et le désir d'aide chez ces femmes afin d'optimiser les soins et d'exercer une incidence positive sur leur qualité de vie.

\section{But}

Le but final de ces travaux est d'améliorer la qualité des soins prodigués aux femmes atteintes d'un cancer de l'ovaire. Le but immédiat de cette étude descriptive transversale était de dégager l'éventail élargi des besoins en soins de soutien éprouvés par les femmes porteuses d'un cancer ovarien et fréquentant un centre de cancérologie ambulatoire intégré. Un but secondaire était de déterminer si les patientes voulaient de l'aide afin de satisfaire ces besoins exprimés.

\section{Méthodologie}

\section{Procédure}

Après avoir reçu l'approbation éthique, nous avons contacté toutes les patientes atteintes d'un cancer ovarien fréquentant la clinique de gynécologie du centre de cancérologie au cours d'une période de quatre mois consécutifs pour voir si elles désiraient participer à l'étude. Cette prise de contact se faisait dans l'aire d'accueil de la clinique et était effectuée par l'assistante de recherche qui expliquait à chaque patiente les objectifs de l'étude et les détails de la participation. Les patientes qui acceptaient d'y participer signaient un formulaire de consentement et recevaient un questionnaire d'autoévaluation à remplir. Si la patiente le remplissait tandis qu'elle attendait le début de son rendez-vous, elle le remettait directement à l'assistante de recherche. Par contre, si la patiente n'avait pas la possibilité de remplir le questionnaire à ce moment-là, l'assistante de recherche donnait à la patiente une enveloppe préadressée et affranchie dans laquelle celle-ci pouvait retourner le questionnaire. Le taux de réponse global s'élevait à 49,3 \%.

\section{Collecte et analyse des données}

Les patientes devaient remplir une fiche démographique comprenant six questions : âge en années; situation maritale (mariée/cohabitation avec un partenaire de vie intime, séparée/divorcée, veuve, célibataire); plus haut niveau de scolarité atteint (aucune instruction scolaire, école primaire, école secondaire, programme collégial, programme universitaire); mois et année du diagnostic de cancer et type de cancer (ovaire, vulve, endomètre, col utérin, utérus, autre type); si la patiente reçoit actuellement un traitement, et dans la négative, le mois et l'année du dernier traitement; et enfin, type de traitement reçu pour le cancer (radiothérapie, chimiothérapie, chirurgie, autre). Pour cette dernière question, les patientes cochaient toutes les réponses qui convenaient.
Les patientes ont également rempli un questionnaire. L'enquête sur les besoins en soins de soutien qui a été utilisée dans le cadre de l'étude est basée sur un instrument qui avait été élaboré et validé en Australie (Bonevski et al., 2000). L'instrument original comportait 60 questions fermées auxquels les patients répondaient en utilisant une échelle de type Likert en 5 points. Selon sa définition, l'échelle reflète divers niveaux de besoin allant de $1=$ "Aucun besoin » (c.-à-d. l'item ne constitue pas un problème pour le patient du fait de son cancer) à 5 = des besoins s'accompagnant d'un " Grand besoin d'aide » (c.-à-d. l'item est un souci de taille ou revêt une grande importance pour le patient, et ce dernier éprouve un grand besoin d'aide supplémentaire face au problème.

L'analyse factorielle en composantes principales effectuée dans l'étude originelle (Bonevski et coll., 2000) a révélé des items regroupés en fonction de cinq facteurs (domaines) : 1) facteurs psychologiques-les items reliés aux émotions et à l'adaptation; 2) système de santé et information dans ce domaine-les items reliés au centre de traitement et l'information concernant la maladie, le traitement et le suivi; 3 ) facteurs physiques et vie quotidienne-les items liés à l'adaptation aux symptômes physiques, aux effets secondaires du traitement et à la réalisation des tâches et activités physiques habituelles; 4) soins et soutien au patient-les items relatifs à la manifestation, par les prestataires de soins, d'une sensibilité aux besoins physiques et affectifs, au respect de la vie privée et à la possibilité de faire des choix; et enfin, 5) sexualité-les items relatifs aux relations sexuelles. Les coefficients alpha de Cronbach associés aux cinq facteurs variaient de 0,87 pour la sexualité à 0,97 pour les aspects psychologiques. Quatre autres items n'entretenaient aucun lien avec un facteur de pondération particulier-le transport, les attitudes d'autrui envers la patiente, les finances et le fait de parler à d'autres personnes.

L'instrument de mesure qui est aisément compris par les individus ayant reçu une éducation minimale se remplit en une vingtaine de minutes (Bonevski et al., 2000). Cet instrument avait été révisé dans notre centre de cancérologie afin qu'il puisse être employé auprès de deux groupes : les patients atteints de cancer du poumon ou ceux atteints d'un cancer avancé qui fréquentaient la clinique de radiothérapie palliative du centre. Les résultats de fidélité étaient encourageants pour les patients atteints d'un cancer du poumon avec des sous-échelles (domaines) comprises entre 0,66 et $0,90,6$ sur 7 d'entre elles se montant à 0,80 ou plus. Pour ce qui est du groupe recevant un traitement palliatif, les coefficients alpha de Cronbach variaient de 0,35 à $0,81,5$ sur 7 d'entre eux étant égaux ou supérieurs à 0,70 . L'essai pilote de l'instrument original nous avait amenées à ordonner différemment certaines questions dans un souci de clarté. De légères modifications terminologiques ont également été nécessaires afin de refléter notre contexte de soins. Par exemple, nous avons remplacé le terme " hôpital » par " centre de cancérologie ». En outre, l'instrument révisé contenait une question supplémentaire sur le désir de recevoir de l'aide. Pour chaque item, les patientes étaient donc priées d'indiquer si elles souhaitaient recevoir de l'aide pour résoudre le besoin (c.-à-d. Non, je ne veux aucune aide; Oui, je veux bien de l'aide; Je suis incertaine quant à vouloir recevoir de l'aide).

Avant d'être utilisé dans le cadre de la présente étude, l'instrument révisé a été légèrement modifié par les cliniciens de la clinique de gynécologie en collaboration avec l'équipe de recherche. Quelques items ont été supprimés et d'autres ajoutés afin que l'instrument soit d'une plus grande pertinence pour la population visée. Par exemple, le terme " toux » a été supprimé tandis que " écoulement vaginal » a été ajouté. L'enquête sur les besoins en soins de soutien ainsi créée comporte 67 items lesquels couvrent les sept domaines des soins de soutien : affectifs (11 items); informationnels (9 items); physiques (symptômes; 14 items); pratiques (8 items); psychologiques (11 items); sociaux (7 items); et enfin, spirituels (7 items). La validité apparente de cette adaptation de l'instru- 
ment a été déterminée par le biais de son acceptabilité par les patientes ciblées par l'étude. Nous étions certaines de la validité de contenu puisque le contenu des questions se rapprochait de celui des questions de l'instrument original et que des spécialistes s'étaient chargés de l'adaptation. Pour cette adaptation à la sphère gynécologique, les coefficients de fidélité de Cronbach pour les domaines de besoin s'échelonnaient de 0,63 à 0,88, un seul domaine se situant en dessous de 0,70 (voir le tableau 1). Comme les travaux auprès de cette population étaient encore préliminaires, l'instrument a été analysé seulement en tant que liste de contrôle. Seuls les questionnaires ne comportant pas plus de trois questions sans réponse ont été inclus dans nos analyses. Aux fins de la présente étude, l'analyse était de nature descriptive et elle se concentrait sur la fréquence des items.

\section{Résultats}

\section{Échantillon}

L'âge moyen des 50 femmes composant l'échantillon de commodité était de 59,4 ans et reflétait en cela la clientèle de la clinique. La plupart des patientes étaient mariées $(n=33)$, deux tiers avaient fait au moins des études collégiales $(\mathrm{n}=30)$, et pour 36 d'entre elles, le diagnostic remontait à deux ans ou moins. Quarante pour cent n'étaient plus sous traitement $(n=20)$. Le traitement le plus courant était la chimiothérapie $(n=39)$, suivi par la chirurgie $(n=38)$ (voir le tableau 2).

\section{Problèmes actuels fréquemment signalés}

Les femmes déclaraient éprouver actuellement des problèmes dans les sept domaines de besoins et pour tous les items sauf un. Ainsi, aucune participante ne signalait de problème au niveau du respect de leur vie privée dans la clinique. Le nombre de patientes éprouvant un problème variait de $\mathrm{n}=1$ (physiques : " écoulement vaginal ») à $n=36$ (psychologiques : " craintes que le cancer ne revienne »). Dans tous les domaines, il y avait au moins un quart des répondantes qui déclaraient éprouver actuellement un problème (voir les tableaux 3 à 9).

Parmi les 10 problèmes actuellement éprouvés que les participantes signalaient le plus fréquemment, trois appartenaient au domaine affectif (sentiments de tristesse, se sentir abattue ou déprimée, s'inquiéter que les résultats du traitement échappent à votre contrôle). Deux étaient d'ordre physique (manque d'énergie, ne pas être capable de faire ce que vous pouviez faire auparavant); deux étaient de nature psychologique (craintes que le cancer ne revienne, craintes que le cancer ne se propage); un d'ordre social (s'inquiéter du souci que se font les personnes qui vous sont chères); un se rapportait au domaine spirituel (incertitude au sujet de l'avenir); et enfin, un avait trait au domaine pratique (changements aux routines et au mode de vie) (voir le tableau 10).

Tableau 1. Enquête sur les besoins en soins de soutiengynécologie : domaines et coefficients de fidélité

\begin{tabular}{|l|l|l|}
\hline Domaines & Nombre d'items & $\begin{array}{l}\text { Coefficients alpha } \\
\text { de Cronbach }\end{array}$ \\
\hline Besoins affectifs & 11 & 0,88 \\
\hline Besoins spirituels & 7 & 0,87 \\
\hline Besoins psychologiques & 11 & 0,85 \\
\hline Symptômes physiques & 14 & 0,85 \\
\hline Besoins d'information & 9 & 0,83 \\
\hline Besoins sociaux & 7 & 0,76 \\
\hline Besoins pratiques & 8 & 0,63 \\
\hline
\end{tabular}

\section{Besoins d'information}

Lors de leur participation à l'enquête, au moins 25 \% des femmes éprouvaient des problèmes relativement à trois des items du domaine de l'information : être informée de ce que vous pouvez faire afin de vous rétablir $(n=15)$, être informée le plus rapidement possible des résultats des examens $(n=14)$, et enfin, recevoir des explications à propos des tests pour lesquels vous souhaitez en avoir $(n=13)$ (voir le tableau 3$)$.

\section{Besoins affectifs}

Au moins $25 \%$ des répondantes avaient actuellement des besoins affectifs relativement à six items : sentiments de tristesse $(n=25)$, s'inquiéter que les résultats du traitement échappent à votre contrôle $(n=24)$, se sentir abattue ou déprimée $(n=23)$, anxiété à l'idée de recevoir un quelconque traitement $(n=22)$, anxiété $(n=20)$, et enfin, sentiments d'ennui et/ou d'inutilité $(\mathrm{n}=17)$ (voir le tableau 4).

\section{Besoins psychologiques}

Les items craintes que le cancer ne revienne $(n=39)$ et craintes que le cancer ne se propage $(n=35)$ se classaient aux deux premiers rangs des besoins globaux et constituaient deux des 6 items psychologiques pour lesquels au moins $25 \%$ des femmes éprouvaient un problème. Les quatre autres étaient les suivants : apprendre à sentir que vous maîtrisez la situation $(n=20)$, craintes relatives à la douleur $(n=20)$, craintes relatives à la déficience ou à la détérioration physique $(n=19)$ et enfin, accepter les changements sur le plan de l'apparence physique $(\mathrm{n}=17)$ (voir le tableau 5).

\section{Besoins physiques}

Plus de $25 \%$ des patientes exprimaient des besoins physiques relativement à six items : manque d'énergie $(n=28)$, ne pas être capable de faire ce que vous pouviez faire auparavant $(n=26)$, malaise abdominal $(\mathrm{n}=22)$, perturbation du transit intestinal $(n=19)$, ne pas être capable de faire les travaux ménagers $(n=15)$, et enfin, perte d'appétit $(\mathrm{n}=14)$ (voir le tableau 6).

\section{Besoins spirituels}

Six items représentaient des problèmes actuels chez au moins un quart des patientes sondées : incertitude au sujet de l'avenir $(n=28)$, émotions relatives à la mort et au mourir $(n=20)$, confusion quant

Tableau 2. Variables démographiques $(n=50 *)$

\begin{tabular}{|l|l|l|}
\hline Âge & $<45$ ans & 12 \\
& $45-65$ ans & 13 \\
& $>65$ ans & 24 \\
\hline $\begin{array}{l}\text { Situation } \\
\text { maritale }\end{array}$ & Mariée & 33 \\
& $\begin{array}{l}\text { Séparée/divorcée/veuve } \\
\text { Célibataire (jamais mariée) }\end{array}$ & 10 \\
\hline $\begin{array}{l}\text { Niveau de } \\
\text { scolarité }\end{array}$ & $\begin{array}{l}\text { Aucune instruction scolaire } \\
<\text { Études collégiales }\end{array}$ & 1 \\
\hline $\begin{array}{l}\text { Temps écoulé } \\
\text { depuis le } \\
\text { diagnostic }\end{array}$ & $\geq$ Études collégiales & 18 \\
\hline $\begin{array}{l}\text { Actuellement } \\
\text { sous traitement }\end{array}$ & $\begin{array}{l}\text { 1-2 an(s) } \\
\text { Oni ans }\end{array}$ & 30 \\
\hline $\begin{array}{l}\text { Traitement } \\
\text { dispensé }\end{array}$ & Non & 28 \\
& Chimiothérapie & 14 \\
\hline Chirurgie & 30 \\
\hline * L'absence de données dans certains cas fait que les totaux ne \\
sont pas toujours égaux à 50.
\end{tabular}


aux raisons pour lesquelles ceci vous est arrivé $(n=19)$, rester positive $(n=15)$, donner un sens à cette expérience $(n=15)$ et enfin, tirer le meilleur parti de votre temps $(\mathrm{n}=14)$ (voir le tableau 7$)$.

\section{Besoins sociaux}

On dénombrait cinq items dans la catégorie des besoins sociaux pour lesquels plus de $25 \%$ des femmes éprouvaient actuellement un problème : s'inquiéter du souci que se font les personnes qui vous sont chères $(n=29)$, s'inquiéter de s'acquitter de son rôle de partenaire de vie $(n=14)$, s'inquiéter de la capacité de vos proches à composer avec votre soin $(\mathrm{n}=19)$, inquiétudes relatives à votre rôle d'aidante naturelle $(n=13)$ et enfin, changements relatifs aux attitudes et comportements d'autrui envers vous $(n=13)$ (voir le tableau 8).

Tableau 3. Besoins d'information $(\mathrm{N}=50)$

Problème

Être informée de ce que vous pouvez faire afin de vous rétablir

Être informée le plus rapidement possible des résultats des examens

Recevoir des explications à propos des tests pour lesquels vous souhaitez en avoir

Être informée de la situation de votre cancer lorsqu'il est maîtrisé ou s'atténue (rémission)

Être informée convenablement des avantages et des effets secondaires du traitement

Être informée des groupes de soutien existant dans votre région

Recevoir de l'information sur les relations sexuelles

Recevoir de l'information écrite concernant des aspects importants de vos soins

Recevoir de l'information sur divers aspects de la gestion de votre maladie et des effets secondaires du traitement

\begin{tabular}{|l|l|l|l|}
\hline $\begin{array}{l}\text { Éprouvent } \\
\text { actuellement } \\
\text { ce problème }\end{array}$ & $\begin{array}{l}\text { Veulent } \\
\text { obtenir } \\
\text { de l'aide }\end{array}$ & $\begin{array}{l}\text { Incertaines } \\
\text { de vouloir } \\
\text { obtenir } \\
\text { de l'aide }\end{array}$ & $\begin{array}{l}\text { Veulent } \\
\text { obtenir de } \\
\text { l'aide ou Sont } \\
\text { incertaines/\%* }\end{array}$ \\
\hline 15 & 18 & 0 & $18 />100 \%$ \\
\hline 14 & 12 & 0 & $12 / 85,7 \%$ \\
\hline 10 & 11 & 0 & $14 />100 \%$ \\
\hline 8 & 9 & 0 & $11 />100 \%$ \\
\hline 6 & 9 & 2 & $9 />100 \%$ \\
\hline 5 & 5 & 2 & $7 />100 \%$ \\
\hline 5 & 11 & 0 & $11 />100 \%$ \\
\hline 5 & 12 & 2 & $14 />100 \%$ \\
\hline
\end{tabular}

incertaines à ce sujet divisé par le nombre total de femmes éprouvant actuellement ce problème; \% > 100 où les femmes ont indiqué qu'elles voulaient de l'aide pour un problème donné alors qu'elles ne l'éprouvaient pas actuellement

\begin{tabular}{|c|c|c|c|c|}
\hline Problème & $\begin{array}{l}\text { Éprouvent } \\
\text { actuellement } \\
\text { ce problème }\end{array}$ & $\begin{array}{l}\text { Veulent } \\
\text { obtenir } \\
\text { de l'aide }\end{array}$ & $\begin{array}{l}\text { Incertaines } \\
\text { de vouloir } \\
\text { obtenir } \\
\text { de l'aide }\end{array}$ & $\begin{array}{l}\text { Veulent } \\
\text { obtenir de } \\
\text { l'aide ou Sont } \\
\text { incertaines/\%* }\end{array}$ \\
\hline Sentiments de tristesse & 25 & 15 & 3 & $18 / 72,0 \%$ \\
\hline S'inquiéter que les résultats du traitement échappent à votre contrôle & 24 & 10 & 0 & $10 / 41,7 \%$ \\
\hline Se sentir abattue ou déprimée & 23 & 14 & 0 & $14 / 60,9 \%$ \\
\hline Anxiété à l'idée de recevoir un quelconque traitement & 22 & 11 & 2 & $13 / 59,1 \%$ \\
\hline Anxiété & 20 & 15 & 1 & $16 / 80,0 \%$ \\
\hline Sentiments d'ennui et/ou d'inutilité & 17 & 10 & 0 & $10 / 62,3 \%$ \\
\hline Avoir un membre du personnel hospitalier à qui parler & 11 & 14 & 1 & $15 />100 \%$ \\
\hline Être traitée comme une personne et non comme un numéro & 10 & 5 & 2 & $7 / 70,0 \%$ \\
\hline Changements au niveau des relations sexuelles & 9 & 4 & 4 & $8 / 88,9 \%$ \\
\hline $\begin{array}{l}\text { La possibilité de parler avec quelqu'un qui comprend et qui a traversé } \\
\text { une expérience similaire }\end{array}$ & 8 & 9 & 1 & $10 />100 \%$ \\
\hline Que le personnel hospitalier reconnaisse vos sentiments et y soit sensible & 6 & 6 & 0 & $6 / 100 \%$ \\
\hline \multicolumn{5}{|c|}{$\begin{array}{l}*=\% \text { calculé comme étant le nombre total de femmes qui souhaitaient recevoir de l'aide pour un problème donné ou } \\
\text { étaient incertaines à ce sujet divisé par le nombre total de femmes éprouvant actuellement ce problème; \% > } 100 \text { où les } \\
\text { femmes ont indiqué qu'elles voulaient de l'aide pour un problème donné alors qu'elles ne l'éprouvaient pas actuellement }\end{array}$} \\
\hline
\end{tabular}




\begin{tabular}{|c|c|c|c|c|}
\hline Problème & $\begin{array}{l}\text { Éprouvent } \\
\text { actuellement } \\
\text { ce problème }\end{array}$ & $\begin{array}{l}\text { Veulent } \\
\text { obtenir } \\
\text { de l'aide }\end{array}$ & $\begin{array}{l}\text { Incertaines } \\
\text { de vouloir } \\
\text { obtenir de } \\
\text { l'aide }\end{array}$ & $\begin{array}{l}\text { Veulent } \\
\text { obtenir de } \\
\text { l'aide ou Sont } \\
\text { incertaines/\%* }\end{array}$ \\
\hline Craintes que le cancer ne revienne & 36 & 17 & 1 & $18 / 50 \%$ \\
\hline Craintes que le cancer ne se propage & 35 & 17 & 6 & $23 / 65,7 \%$ \\
\hline Apprendre à sentir que vous maîtrisez la situation & 20 & 10 & 2 & $12 / 60 \%$ \\
\hline Craintes relatives à la douleur & 20 & 12 & 3 & $15 / 75,0 \%$ \\
\hline Craintes relatives à la déficience ou à la détérioration physique & 19 & 10 & 1 & $11 / 57,9 \%$ \\
\hline Accepter les changements sur le plan de l'apparence physique & 17 & 7 & 1 & $8 / 47,1 \%$ \\
\hline $\begin{array}{l}\text { Avoir accès à des consultations professionnelles si vous ou vos } \\
\text { proches en avez besoin }\end{array}$ & 10 & 9 & 3 & $12 />100 \%$ \\
\hline Craintes relatives à la perte d'autonomie & 9 & 9 & 1 & $10 />100 \%$ \\
\hline $\begin{array}{l}\text { Être rassurée par le personnel médical que ce que vous } \\
\text { ressentez est normal }\end{array}$ & 7 & 8 & 0 & $8 />100 \%$ \\
\hline Être traitée dans une clinique où l'ambiance est plaisante & 5 & 6 & 0 & $6 />100 \%$ \\
\hline $\begin{array}{l}\text { Que vos droits au respect de votre vie privée soient mieux protégés } \\
\text { lorsque vous êtes à la clinique }\end{array}$ & 0 & 0 & 0 & $0 / 0 \%$ \\
\hline \multicolumn{5}{|c|}{$\begin{array}{l}* \text { \% calculé comme étant le nombre total de femmes qui souhaitaient recevoir de l'aide pour un problème donné ou étaient } \\
\text { incertaines à ce sujet divisé par le nombre total de femmes éprouvant actuellement ce problème; \% > } 100 \text { où les femmes ont } \\
\text { indiqué qu'elles voulaient de l'aide pour un problème donné alors qu'elles ne l'éprouvaient pas actuellement }\end{array}$} \\
\hline
\end{tabular}

Tableau 6. Besoins physiques $(\mathrm{N}=50)$

\begin{tabular}{|c|c|c|c|c|}
\hline Problème & $\begin{array}{l}\text { Éprouvent } \\
\text { actuellement } \\
\text { ce problème }\end{array}$ & $\begin{array}{l}\text { Veulent } \\
\text { obtenir } \\
\text { de l'aide }\end{array}$ & $\begin{array}{l}\text { Incertaines } \\
\text { de vouloir } \\
\text { obtenir de } \\
\text { l'aide }\end{array}$ & $\begin{array}{l}\text { Veulent } \\
\text { obtenir de } \\
\text { l'aide ou Sont } \\
\text { incertaines/\%" }\end{array}$ \\
\hline Manque d'énergie & 28 & 12 & 3 & $15 / 53,6 \%$ \\
\hline Ne pas être capable de faire ce que vous pouviez faire auparavant & 26 & 7 & 5 & $12 / 46,2 \%$ \\
\hline Malaise abdominal & 22 & 10 & 4 & $14 / 63,6 \%$ \\
\hline Perturbation du transit intestinal & 19 & 9 & 3 & $12 / 63,2 \%$ \\
\hline Ne pas être capable de faire les travaux ménagers & 15 & 3 & 2 & $5 / 33,3 \%$ \\
\hline Perte d'appétit & 14 & 4 & 2 & $6 / 42,9 \%$ \\
\hline Changements au niveau de la libido & 11 & 5 & 4 & $9 / 81,8 \%$ \\
\hline Changements au niveau de votre aptitude à avoir des rapports sexuels & 11 & 4 & 4 & $8 / 72,7 \%$ \\
\hline Perturbation de la fonction urinaire & 11 & 5 & 2 & $7 / 63,6 \%$ \\
\hline Douleur & 9 & 6 & 1 & $7 / 77,8 \%$ \\
\hline CEdème aux jambes & 7 & 2 & 0 & $2 / 28,6 \%$ \\
\hline Nausée/vomissements & 7 & 1 & 2 & $3 / 42,9 \%$ \\
\hline $\begin{array}{l}\text { Que le personnel du centre s'occupe promptement de } \\
\text { vos besoins physiques }\end{array}$ & 6 & 5 & 0 & $5 / 83,3 \%$ \\
\hline Écoulement vaginal & 1 & 1 & 0 & $1 / 100 \%$ \\
\hline
\end{tabular}




\section{Besoins pratiques}

En ce qui concerne cette dernière dimension, au moins $25 \%$ des patientes ont exprimé des besoins actuels par rapport à seulement deux items : changements aux routines et au mode de vie $(n=25)$ et devoir attendre longtemps lors des rendez-vous cliniques $(n=15)$ (voir le tableau 9).

\section{Détresse autodéclarée relative à un problème actuel}

Les patientes qui éprouvaient actuellement un problème étaient également invitées à préciser l'ampleur de la détresse qu'elles ressentaient du fait de ce problème. Elles indiquaient si la détresse se rapportant à chacun des 10 items les plus fréquemment sélectionnés était d'une intensité faible, modérée ou élevée (voir le tableau 10). Il convient de remarquer que pour 7 des 10 items, une détresse élevée était mentionnée par au moins un quart des répondantes éprouvant le problème en question.

\section{Aide souhaitée}

Les patientes éprouvant actuellement un problème étaient également priées d'indiquer si elles souhaitaient recevoir de l'aide pour résoudre ledit besoin. Nous avons posé l'hypothèse que toute personne qui se disait « incertaine » méritait, au minimum, une con- versation avec le personnel afin d'explorer un besoin d'aide éventuel ou réel. Il s'ensuit que les réponses des participantes qui se sont déclarées « incertaines » quant à leur désir de recevoir de l'aide ont été ajoutées à celles des répondantes qui avaient indiqué vouloir recevoir de l'aide. Le total ainsi obtenu a été divisé par le nombre de répondantes ayant signalé qu'elles éprouvaient actuellement le problème. Ces calculs ont permis de déterminer les pourcentages des patientes éprouvant actuellement un problème qui désiraient un quelconque type d'aide. Toutefois, dans un certain nombre de cas, il y avait davantage de femmes qui indiquaient vouloir recevoir de l'aide que de femmes ayant signalé que l'item représentait un problème actuel pour elles. Les résultats concernant les répondantes ayant choisi les réponses « oui » ou « incertaine » sont présentés dans les tableaux 3 à 9 .

Un pourcentage élevé des femmes incluses dans cette étude voulaient recevoir de l'aide pour les problèmes qu'elles éprouvaient actuellement. Pour les 67 items excepté les 10 suivants (que vos droits au respect de votre vie privée soient mieux protégés lorsque vous êtes à la clinique, ne pas être capable de faire les travaux ménagers, perte d'appétit, œdème aux jambes, nausée/vomissements, incertitude au sujet de l'avenir, changements relatifs aux attitudes et comportements d'autrui envers vous,

\begin{tabular}{|c|c|c|c|c|}
\hline Problème & $\begin{array}{l}\text { Éprouvent } \\
\text { actuellement } \\
\text { ce problème }\end{array}$ & $\begin{array}{l}\text { Veulent } \\
\text { obtenir } \\
\text { de l'aide }\end{array}$ & $\begin{array}{l}\text { Incertaines } \\
\text { de vouloir } \\
\text { obtenir de } \\
\text { l'aide }\end{array}$ & $\begin{array}{l}\text { Veulent } \\
\text { obtenir de } \\
\text { l'aide ou Sont } \\
\text { incertaines/\%: }\end{array}$ \\
\hline Incertitude au sujet de l'avenir & 28 & 13 & 1 & $14 / 50 \%$ \\
\hline Émotions relatives à la mort et au mourir & 20 & 8 & 6 & $14 / 70,0 \%$ \\
\hline Confusion quant aux raisons pour lesquelles ceci vous est arrivé & 19 & 8 & 3 & $11 / 57,9 \%$ \\
\hline Rester positive & 15 & 10 & 2 & $12 / 80,0 \%$ \\
\hline Donner un sens à cette expérience & 15 & 7 & 2 & $9 / 60,0 \%$ \\
\hline Tirer le meilleur parti de votre temps & 14 & 9 & 2 & $11 / 78,6 \%$ \\
\hline Que le personnel clinique soit source d'espoir & 11 & 5 & 1 & $6 / 54,5 \%$ \\
\hline
\end{tabular}

\begin{tabular}{|c|c|c|c|c|}
\hline Problème & $\begin{array}{l}\text { Éprouvent } \\
\text { actuellement } \\
\text { ce problème }\end{array}$ & $\begin{array}{l}\text { Veulent } \\
\text { obtenir } \\
\text { de l'aide }\end{array}$ & $\begin{array}{l}\text { Incertaines } \\
\text { de vouloir } \\
\text { obtenir de } \\
\text { l'aide }\end{array}$ & $\begin{array}{l}\text { Veulent } \\
\text { obtenir de } \\
\text { l'aide ou Sont } \\
\text { incertaines/\%* }\end{array}$ \\
\hline S'inquiéter du souci que se font les personnes qui vous sont chères & 29 & 11 & 6 & $17 / 58,6 \%$ \\
\hline S’inquiéter de la capacité de vos proches à composer avec votre soin & 19 & 9 & 4 & $13 / 68,4 \%$ \\
\hline S’inquiéter de s'acquitter de son rôle de partenaire de vie & 14 & 8 & 2 & $10 / 71,4 \%$ \\
\hline Inquiétudes relatives à votre rôle d'aidante naturelle & 13 & 8 & 3 & $11 / 84,6 \%$ \\
\hline Changements relatifs aux attitudes et comportements d'autrui envers vous & 13 & 6 & 0 & $6 / 46,2 \%$ \\
\hline Parler du cancer avec autrui & 3 & 4 & 1 & $5 />100 \%$ \\
\hline $\begin{array}{l}\text { Que vos proches et amis puissent être avec vous à la clinique chaque } \\
\text { fois que vous le désirez }\end{array}$ & 2 & 0 & 0 & $0 / 0 \%$ \\
\hline \multicolumn{5}{|c|}{$\begin{array}{l}\text { * = \% calculé comme étant le nombre total de femmes qui souhaitaient recevoir de l'aide pour un problème donné ou étaient } \\
\text { incertaines à ce sujet divisé par le nombre total de femmes éprouvant actuellement ce problème; \% > } 100 \text { où les femmes ont indiqué } \\
\text { qu'elles voulaient de l'aide pour un problème donné alors qu'elles ne l'éprouvaient pas actuellement }\end{array}$} \\
\hline
\end{tabular}


accepter les changements sur le plan de l'apparence physique, ne pas être capable de faire ce que vous pouviez faire auparavant, changements aux routines et au mode de vie et enfin, s'inquiéter que les résultats du traitement échappent à votre contrôle), au moins 50 \% des patients éprouvant un besoin donné voulaient avoir de l'aide pour le satisfaire. Les trois derniers items se classaient aux septième, huitième et neuvième rangs, respectivement, des dix problèmes les plus fréquemment éprouvés, mais un peu moins de $50 \%$ des femmes éprouvant un besoin dans ces domaines souhaitaient recevoir de l'aide. Il est intéressant de noter que bien que peu de femmes éprouvaient des besoins d'information, quasiment toutes les répondantes éprouvant un besoin dans ce domaine voulaient qu'on les aide à son sujet. Il est plutôt surprenant de constater que pour 8 sur 9 des items sur l'information, plus de femmes signalaient vouloir de l'aide au niveau d'un problème donné qu'il n'y en avait qui déclaraient éprouver actuellement des inquiétudes à son sujet. La même constatation a été faite relativement à trois items psychologiques, à deux items affectifs et à un item social.

\section{Discussion}

Cette étude transversale a été entreprise afin de décrire la gamme complète des besoins en soins de soutien des femmes atteintes d'un cancer de l'ovaire. Quoique l'étude a été réalisée dans un seul contexte de soins et n'a obtenu qu'un taux de réponse raisonnable, les données fournissent un portrait initial de l'ampleur éventuelle des besoins chez cette clientèle à un moment donné. Il est évident que les besoins des femmes à l'étude dépassaient le seul domaine physique et qu'elles éprouvaient simultané-

\begin{tabular}{|c|c|c|c|c|}
\hline Problème & $\begin{array}{l}\text { Éprouvent } \\
\text { actuellement } \\
\text { ce problème }\end{array}$ & $\begin{array}{l}\text { Veulent } \\
\text { obtenir } \\
\text { de l'aide }\end{array}$ & $\begin{array}{l}\text { Incertaines } \\
\text { de vouloir } \\
\text { obtenir de } \\
\text { l'aide }\end{array}$ & $\begin{array}{l}\text { Veulent } \\
\text { obtenir de } \\
\text { l'aide ou Sont } \\
\text { incertaines/\%* }\end{array}$ \\
\hline Changements aux routines et au mode de vie & 25 & 12 & 0 & $12 / 48,0 \%$ \\
\hline Devoir attendre longtemps lors des rendez-vous cliniques & 15 & 7 & 1 & $8 / 53,3 \%$ \\
\hline Inquiétudes relatives à votre situation financière & 10 & 6 & 3 & $9 / 90 \%$ \\
\hline $\begin{array}{l}\text { Inquiétudes relatives aux frais financiers associés à vos médicaments } \\
\text { sur ordonnance }\end{array}$ & 8 & 5 & 2 & $7 / 87,5 \%$ \\
\hline Avoir des choix quant aux rendez-vous pour les tests et les traitements & 8 & 7 & 1 & $8 / 100 \%$ \\
\hline $\begin{array}{l}\text { Inquiétudes relatives aux déplacements à destination et en provenance } \\
\text { de la clinique }\end{array}$ & 4 & 2 & 1 & $3 / 75,0 \%$ \\
\hline Plus de choix quant à la clinique où vous devez aller pour vous faire soigner & 2 & 1 & 0 & $1 / 50 \%$ \\
\hline \multicolumn{5}{|c|}{$\begin{array}{l}*=\% \text { calculé comme étant le nombre total de femmes qui souhaitaient recevoir de l'aide pour un problème donné ou étaient } \\
\text { incertaines à ce sujet divisé par le nombre total de femmes éprouvant actuellement ce problème; \%>100 où les femmes ont indiqué } \\
\text { qu'elles voulaient de l'aide pour un problème donné alors qu'elles ne l'éprouvaient pas actuellement }\end{array}$} \\
\hline
\end{tabular}

Tableau 10. Items les plus fréquemment déclarés, tous domaines de soins de soutien confondus

\begin{tabular}{|c|c|c|c|c|c|c|}
\hline \multirow[t]{2}{*}{ Problème } & \multirow{2}{*}{$\begin{array}{l}\text { Éprouvent } \\
\text { actuellement } \\
\text { ce problème }\end{array}$} & \multirow{2}{*}{$\begin{array}{l}\text { Veulent } \\
\text { obtenir } \\
\text { de l'aide }\end{array}$} & \multirow{2}{*}{$\begin{array}{l}\text { Incertaines de } \\
\text { vouloir obtenir } \\
\text { de l'aide }\end{array}$} & \multicolumn{3}{|c|}{$\begin{array}{l}\text { Degré de détresse } \\
\text { causé par le problème }\end{array}$} \\
\hline & & & & Faible & Mod. & Élevée \\
\hline Craintes que le cancer ne revienne (PSY) & 36 & 17 & 7 & 12 & 15 & $9 *$ \\
\hline Craintes que le cancer ne se propage (PSY) & 35 & 17 & 6 & 15 & 11 & $9 *$ \\
\hline $\begin{array}{l}\text { S'inquiéter du souci que se font les personnes qui } \\
\text { vous sont chères (SOC) }\end{array}$ & 29 & 11 & 6 & 11 & 12 & 6 \\
\hline Incertitude au sujet de l'avenir (SP) & 28 & 13 & 1 & 14 & 7 & $7^{*}$ \\
\hline Manque d'énergie (PH) & 28 & 12 & 3 & 11 & 10 & $7^{*}$ \\
\hline $\begin{array}{l}\text { Ne pas être capable de faire ce que vous pouviez } \\
\text { faire auparavant }(\mathrm{PH})\end{array}$ & 26 & 7 & 5 & 10 & 10 & 6 \\
\hline Sentiments de tristesse (AFF) & 25 & 15 & 3 & 12 & 8 & 5 \\
\hline Changements aux routines et au mode de vie (PR) & 25 & 12 & 0 & 12 & 6 & $7 *$ \\
\hline $\begin{array}{l}\text { S’inquiéter que les résultats du traitement } \\
\text { échappent à votre contrôle (AFF) }\end{array}$ & 24 & 10 & 0 & 13 & 5 & $6^{*}$ \\
\hline Se sentir abattue ou déprimée (AFF) & 23 & 14 & 0 & 10 & 7 & $6^{*}$ \\
\hline
\end{tabular}


ment tout un éventail de problèmes. De plus, une proportion importante d'entre elles vivait une grande détresse à cause des problèmes qu'elles tentaient de gérer.

Il n'est guère surprenant que les 10 principaux problèmes incluent des besoins affectifs (p. ex. Norton et al., 2005; Zabora et al., 2001). En particulier, le sentiment de perte de contrôle ou de n'avoir aucun contrôle est constaté par Norton et al. qui émettaient l'hypothèse qu'une réduction perçue du contrôle est corrélée à une augmentation de la détresse psychologique. Et pourtant, les systèmes de soins de santé ont tendance à renforcer cette perte de contrôle en exigeant des patients qu'ils attendent des heures lors de leurs rendez-vous ou en ne leur offrant aucun choix quant au lieu et à la date où auront lieu leurs examens et traitements. Un modèle de soins axés sur les patients préconisant que les patientes et les prestataires de soins travaillent en équipe pourrait aider à atténuer une partie des inquiétudes actuelles relatives au manque de contrôle.

Le manque d'énergie (ou la fatigue) a été mentionné par d'autres chercheurs (p. ex. Donovan \& Ward, 2005; Ferrell, Smith, Cullinane et al., 2003). Il existe pourtant une telle masse de résultats probants sur la fatigue et sa gestion que les prestataires devraient suggérer aux patients ce qu'il convient de faire en vue de gérer ce symptôme, étant donné que les femmes peuvent ressentir une grande détresse si elles manquent d'énergie pour s'acquitter de leurs rôles habituels (Ferrell, Smith, Juarez et al., 2003). Il faut donc améliorer la communication dans ce domaine et poursuivre les travaux de recherche permettant d'élaborer et de mettre à l'essai des interventions visant à diminuer la fatigue liée au cancer.

Un résultat intéressant bien qu'inattendu était le nombre de fois où plus de femmes voulaient de l'aide relativement à un problème qu'elles étaient à l'éprouver actuellement. Il est possible qu'il régnait une certaine confusion parmi les répondantes ou que ces dernières avaient mal lu le questionnaire, mais les chercheuses n'avaient pas observé cette divergence lorsqu'elles avaient employé l'instrument auprès de deux autres clientèles du même centre de cancérologie. Il se peut que les résultats aient été influencés par la composition de ce groupe (c.-à-d. uniquement des femmes). Ekwall, Ternestedt et Sorbe (2003) ont écrit que l'information et un dialogue continu avec les prestataires de soins revêtaient une très grande importance pour les femmes atteintes d'un cancer gynécologique. Le besoin d'information a également été signalé par Browall et al. (2004) ainsi que Jefferies (2002). Comme Beever (2004) l'a fait remarquer, « les patients ont besoin d'information pour diverses de raisons » (p. 193) [traduction libre] parce que le cancer et son traitement ont des impacts physiques, sociaux, psychologiques, affectifs, pratiques et spirituels lesquels exigent tous une forme quelconque d'information afin que les patients puissent gérer leur vie. Les interventions futures devraient être conçues de manière à aborder tous les domaines (Otis-Green et al., 2008; Power et al., 2008).

Une proportion appréciable des femmes sont déprimées et ont besoin d'aide à ce sujet. Mais elles peuvent avoir besoin de différentes interventions en fonction de l'ampleur de leur détresse (Zabora et al., 2001). Il est nécessaire d'identifier tôt les patientes à risque élevé afin de mettre en place les interventions prospectives permettant de prévenir la détresse (Schulmann-Green et al., 2008). À la différence de l'instrument utilisé dans cette étude, un bref instrument de dépistage serait mieux accepté à la fois par les patientes et par les prestataires à titre de première étape. Une évaluation plus poussée pourrait être effectuée ultérieurement en fonction des résultats de dépistage. Peu importe les approches retenues, elles doivent pouvoir être utilisées au sein d'un milieu clinique fort occupé.

Il se peut que le personnel doive adopter une approche en vertu de laquelle il demande à chaque cliente lorsqu'elle est dans la salle d'attente d'indiquer le principal problème qu'elle souhaite aborder durant la visite plutôt que de suivre le programme prévu par le ou la prestataire; en effet, ce que le personnel juge important peut ne pas correspondre au problème le plus urgent aux yeux de la cliente. Une telle approche permettrait aux patientes de réfléchir attentivement avant de pénétrer dans la salle d'examen et de ne pas perdre de vue leurs préoccupations principales. La technologie informatique a été utilisée avec succès dans certains milieux afin de promouvoir la détermination des problèmes (Carlsson \& Bultz, 2003; Linden, Yi, Barroetavena, MacKenzie \& Doll, 2005). Par exemple, on met à la disposition des patients une liste de problèmes/enjeux à l'écran d'ordinateurs situés dans la salle d'attente et les patients remplissent la liste au moyen d'un écran tactile avant leur rendezvous. Les résultats sont fournis instantanément à l'équipe de soins qui s'en sert pour offrir des interventions pertinentes après s'être entretenue avec les patients. Il est essentiel que les interventions soient dispensées lors de la visite des femmes à la clinique. Les milieux de pratique doivent donc réfléchir à la manière dont ils fournissent actuellement les soins et y apporter les modifications appropriées (p. ex. un changement au niveau de la répartition de leurs effectifs) afin de garantir la mise en œuvre réussie d'une pratique de ce genre.

Les besoins psychosociaux figuraient aux premiers rangs des problèmes déclarés par les participantes à cette étude, à la fois parce qu'elles les éprouvent actuellement et qu'elles veulent de l'aide à leur sujet. Ce résultat suggère que la pratique actuelle permet de soigner les problèmes physiques mais que d'autres volets des soins de soutien exigent davantage d'attention. Une bonne partie des principaux problèmes étaient d'ordre psychosocial et il se peut que ces domaines ne soient pas évalués pour diverses raisons. Par exemple, les brèves visites à la clinique des patients ambulatoires concentrent surtout l'attention sur les aspects physiques et il se peut que le personnel n'ait pas le temps ou pense qu'il n'a pas le temps d'approfondir des enjeux psychosociaux qui ne manquent jamais d'être complexes et qui requièrent d'excellentes compétences cliniques. Les milieux cliniques étant fort occupés et les pénuries de personnel étant d'actualité, le personnel doit faire preuve d'ingéniosité et d'innovation dans la manière dont il fournit information et soins aux patientes. Par exemple, il pourrait considérer la possibilité de fournir l'information au moyen d'ordinateurs ou de vidéos dans la clinique, ou bien d'établir des procédures permettant aux patients de demander et de recevoir des renseignements ou des conseils par le biais du courrier électronique ou d'Internet. Dans tous les cas, il faut qu'il y ait un juste équilibre entre dispenser de l'information et offrir le genre d'information et de soins personnalisés qui correspondent le mieux aux besoins d'un patient donné.

Il est manifeste qu'il convient d'aborder la gamme complète des besoins en soins de soutien et que la pratique exige un travail de collaboration efficace de la part de l'équipe interprofessionnelle. Jusqu'à présent, il n'existe qu'un petit nombre de modèles de soins pour les milieux de pratique intégrant avec succès l'éventail complet des disciplines. De plus, les pratiques en matière de référence sont loin d'avoir atteint leur efficacité optimum, ce qui signifie que les patients peuvent avoir de la difficulté à accéder aux services appropriés. Il convient de mettre en œuvre des processus afin que les mécanismes de référence aillent bien au-delà de la seule équipe des soins en cancérologie et qu'ils incorporent les spécialistes des disciplines psychosociales, par exemple, et que cela se fasse le plus facilement possible. Enfin, les programmes des grands établissements doivent établir des liens avec des programmes communautaires existants afin que les patients disposent de soins optimaux tout au long de la trajectoire du cancer.

La quantification des besoins en soins de soutien est un nouveau domaine de recherche. Quoique des travaux de recherche qualitative aient déjà été effectués (p. ex. Ekman et al., 2004; Ferrell, Smith, Cullinane et al., 2003; Power et al., 2008), il n'existe qu'un minuscule corpus de recherche quantitative, notamment sur 
le désir d'aide de la part des patients en vue de satisfaire leurs besoins. Cette étude initiale est utile parce qu'elle permet de repérer les problèmes critiques et de dégager les domaines à cibler pour les futurs travaux de recherche et l'élaboration d'interventions. Par exemple, auprès de qui les femmes veulent-elles recevoir de l'aide? Quelle sorte d'aide désirent-elles obtenir? Pourquoi certaines d'entre elles ne souhaitent pas recevoir d'aide? Obtiennent-elles une aide de l'extérieur et dans l'affirmative, auprès de qui? Croient-elles qu'il n'y a rien qui puisse être fait? Les équipes de recherche doivent poser aux femmes ces questions et d'autres du même genre dans leurs travaux à venir. Il se peut qu'il faille adopter différentes approches de prestation des soins en fonction du sexe des patients, et les cliniciens ont besoin de données probantes afin de concevoir les interventions qui s'imposent.

\section{Références}

Andersen, B.L, \& Van Der Does, J. (1994). Surviving gynecologic cancer and coping with sexual morbidity: An international problem. International Journal of Gynecologic Cancer, 4, 225-240.

Armstrong, D.K. (2002). Relapsed ovarian cancer: Challenges and management strategies for a chronic disease. Oncologist, 7(Suppl. 5), 20-28.

Beever, K. (2004). Meeting the information needs of people with cancer [Editorial]. European Journal of Oncology Nursing, 8, 193-194.

Bezjak, A., Tu, D., Bacon, M., Isoba, D., Zee, B., Stuart, G., et al. (2004). Quality of life in ovarian cancer patients. Journal of Clinical Oncology, 22, 4595-4603.

Bodurka-Bevers, D., Basen-Engquist, K., Carmack, C.L., Fitzgerald, M.A., Wolf, J.K., de Morr, C., et al. (2000). Depression, anxiety and quality of life in patients with epithelial ovarian cancer. Gynecologic Oncology, 78, 302-308.

Bonevski, B., Sanson-Fisher, R., Girgis, A., Burton, L., Cook, P., Boyes, A., et al. (2000). Evaluation of an instrument to assess the needs of patients with cancer. Cancer, 88(1), 217-225.

Browall, M., Carlsson, M., \& Horvath, G. (2004). Information needs of women with recently diagnosed ovarian cancer-A longitudinal study. European Journal of Oncology Nursing, 8, 200-207.

Carlsson, L.E., \& Bultz, B.D. (2003). Cancer distress screening: Needs, models and methods. Psychosomatic Research, 55(5), 403-409.

Donovan, H., \& Ward, S. (2005). Representations of fatigue in women receiving chemotherapy for gynecologic cancers. Oncology Nursing Forum, 32(1), 113-116.

Ekman, I., Bergbom, I., Ekman, T., Berthold, H., \& Mahsneh, S. (2004). Maintaining normality and support are central issues when receiving chemotherapy for ovarian cancer. Cancer Nursing, 27(3), 177-182.

Ekwall, E., Ternestedt, B., \& Sorbe, B. (2003). Important aspects of health care for women with gynecologic cancer. Oncology Nursing Forum, 30(2), 313-319.

Ell, K., Sanchez, K., Vourlekis, B., Lee, P., Dwight-Johnson, M., Lagomasino, I., et al. (2005). Depression, correlates of depression, and receipt of depression care among low-income women with breast or gynecologic cancer. Journal of Clinical Oncology, 23, 3052-3060.

Ersek, M., Ferrel, B.R., Dow, K.H., \& Melancon, C.H. (1997). Quality of life in women with ovarian cancer. Western Journal of Nursing Research, 19, 334-350.

Ferrell, B., Cuillinane, C.A., Ervine, K., Melancon, C., Uman, G.C., \& Juarez, G. (2005). Perspectives on the impact of ovarian cancer: Women's view of quality of life. Oncology Nursing Forum, 32, 1143-1149.

\section{Conclusion}

Les femmes atteintes d'un cancer de l'ovaire peuvent avoir besoin d'aide relativement à divers besoins insatisfaits. Ces derniers devraient être dégagés précocement afin que des interventions puissent être mises en place aussi tôt que possible. Alors que le manque d'énergie et le fait d'être incapables de faire ce qu'elles pouvaient faire auparavant représentaient des problèmes pour de nombreuses femmes, la majorité des principaux problèmes dégagés étaient de nature psychosociale. Il est nécessaire de mettre en œuvre les structures et processus qui assureront un repérage aisé et rapide des besoins insatisfaits pouvant être suivi d'une intervention adéquate et immédiate. Il est donc essentiel de lancer de nouvelles recherches afin de dégager les besoins et le type d'aide désiré par les femmes et de mettre au point les interventions appropriées.

Ferrell, B., Smith, S., Cullinane, C., \& Melancon, C. (2003). Symptom concerns of women with ovarian cancer. Journal of Pain and Symptom Management, 25(6), 528-538.

Ferrell, B., Smith, S., Juarez, G., \& Melancon, C. (2003). Meaning of illness and spirituality in ovarian cancer survivors. Oncology Nursing Forum, 30(2), 249-257.

Fish, L.S., \& Lewis, B.E. (1999). Quality of life issues in the management of ovarian cancer. Seminars in Oncology, 25(Suppl. 1), 32-39.

Fitch, M.I., Deane, K.A. \& Howell, D. (2003). Le vécu du cancer ovarien: les perspectives des femmes sur le traitement et la prise de décision thérapeutique. Revue canadienne de soins infirmiers en oncologie, 13(1), 14-20.

Fitch, M.I., Gray, R.E., \& Franssen, E. (2001). Perspectives on living with ovarian cancer: Older women's views. Oncology Nursing Forum, 28, 1433-1442.

Greimel, E.R., \& Freidl, W. (2000). Functioning in daily living and psychological well-being of female patients. Journal of Psychosomatic Obstetrics and Gynecology, 21, 25-30.

Guidozzi, F. (1993). Living with ovarian cancer. Gynecologic Oncology, 50, 202-207.

Hamilton, A.B. (1999). Psychological aspects of ovarian cancer. Cancer Investigation, 17, 335-341.

Hoskins, W.J., Pesez, C.A., Young, R.C., Baraket, P.R., Marlnan, J., \& Randall, M.W. (2005). Principles and practices of gynecologic oncology (4th ed.). Toronto, ON: Lippincott, Williams \& Wilkin.

Howell, D., Fitch, M.I., \& Deane, K. (2003a). Impact of ovarian cancer perceived by women. Cancer Nursing, 26(1), 1-9.

Howell, D., Fitch, M.I., \& Deane, K. (2003b). Women's experiences with recurrent ovarian cancer. Cancer Nursing, 26(1), 10-17.

Institut national du cancer du Canada. (2008). Statistiques canadiennes sur le cancer 2008. Toronto, ON : auteur.

Jefferies, H. (2002). Ovarian cancer patients: Are their informational and emotional needs being met? Journal of Clinical Nursing, 11(1), 41-47.

Jenkins, B. (1998). Patient reports of sexual changes after treatment for gynecological cancer. Oncology Nursing Forum, 15(3), 231-242.

Kornblith, A.B., Thater, H.T., Wong, G., Vlamis, V., Lepore, J.M., Loseth, D.B., et al. (1995). Quality of life of women with ovarian cancer. Gynecologic Oncology, 59, 231-242.

Linden, W., Yi, D., Barroetavena, M.C., MacKenzie, R., \& Doll, R. (2005). Development and validation of a psychosocial screening instrument for cancer. Health Quality of Life Outcomes, 3, 54.

Lockwood-Rayermann, S. (2006). Survivorship issues in ovarian cancer: A review. Oncology Nursing Forum, 33, 553-562. 
Lutgendorf, S., Anderson, B., Ullrich, P., Johnsen, E., Buller, R., Sood, A., et al. (2002). Quality of life and mood in women with gynecologic cancer. Cancer, 94, 131-140.

Montazeri, A., McEwen, J., \& Gillis, C.R. (1996). Quality of life with ovarian cancer: Current state of the research. Supportive Cancer Care, 4, 169-179.

Norton, T., Manne, S., Rubin, S., Carlson, J., Hernandez, E., Edelson, M., et al. (2004). Prevalence and predictors of psychological distress among women with ovarian cancer. Journal of Clinical Oncology, 22, 919-926.

Norton, T., Manne, S., Rubin, S., Hernandez, E., Carlson, J., Bergman, C., et al. (2005). Ovarian cancer patients' psychological distress: The role of physical impairment, perceived unsupportive family and friend behaviours, perceived control, and self-esteem. Health Psychology, 24(2), 143-152.

Otis-Green, S., Ferrel, B., Sun, V., Spolum, M., Morgan, R., \& MacDonald, D. (2008). Feasibility of an ovarian cancer quality of life psychoeducational intervention. Journal of Cancer Education, 23(4), 214-221.

Peterson, R.W., Graham, G., \& Qunlivan, J.A. (2005). Psychologic changes after a gynecologic cancer. Journal of Obstetrics and Gynecologic Research, 31(2), 152-157.

Pignata, S., Ballatori, E., Favalli, G., \& Scambia, G. (2001). Quality of life: Gynecological cancers. Annals of Oncology, 12(3), 37-42.
Portenoy, R.K., Kornblith, A.B., Wong, G., Vlamis, Y., Lepore, J.M., Loseth, D.B., et al. (1994). Pain in ovarian cancer: Prevalence, characteristics, and associated symptoms. Cancer, 74(3), 907-915.

Power, J., Brown, L., \& Ritvo, P. (2008). A qualitative study examining psychosocial distress, coping and social support across the stages and phases of epithelial ovarian cancer. Health Care for Women International, 29(4), 366-383.

Robinson, G.E., Rosen, B.P., \& Bradley, L.N. (1997). Psychological impact of screening for familial ovarian cancer: Reactions to initial assessment. Gynecologic Oncology, 65, 197-205.

Schulman-Green, D., Ercolano, E., Dowd, M., Schwartz, P., \& McCorkle, R. (2008). Quality of life among women after surgery for ovarian cancer. Palliative and Supportive Care, 6(3), 239-247.

Steele, R., \& Fitch, M.I. (2008). Supportive care needs of women with gynecologic cancer. Cancer Nursing, 31(4), 284-291.

Tobano, M., Condosta, D., \& Coons, M. (2002). Symptoms affecting the quality of life in women with gynecologic cancer. Seminars in Oncology Nursing, 18(3), 223-230.

Wenzel, L.B., Donnelly, J.P., Fowler, J.M., Habbal, R., Taylor, T.H., Aziz, N., et al. (2002). Resilience, reflection, and residual stress in ovarian cancer survivorship. Psycho-Oncology, 11, 142-154.

Zabora, J., Brintzenhofeszoc, K., Curbow, B., Hooker, C., \& Piantadosi, S. (2001). The prevalence of psychological distress by cancer site. Psycho-Oncology, 10, 19-28. 